\title{
A dynamic interplay between alternative polyadenylation and microRNA regulation: Implications for cancer (Review)
}

\author{
JINDAN AN ${ }^{1,2}$, XIAODONG ZHU ${ }^{1}$, HONGWEI WANG ${ }^{2}$ and XIUDONG JIN ${ }^{1}$ \\ ${ }^{1}$ Key Laboratory of Cancer Prevention and Treatment of Heilongjiang Province, \\ ${ }^{2}$ Department of Pathology, Mudanjiang Medical University, Mudanjiang, P.R. China
}

Received June 14, 2013; Accepted July 18, 2013

DOI: $10.3892 /$ ijo.2013.2047

\begin{abstract}
Alternative polyadenylation and microRNA regulation are both mechanisms of post-transcriptional regulation of gene expression. Alternative polyadenylation often results in mRNA isoforms with the same coding sequence but different lengths of 3' UTRs, while microRNAs regulate gene expression by binding to specific mRNA 3' UTRs. In this sense, different isoforms of an mRNA may be differentially regulated by microRNAs, sometimes resulting in cellular proliferation and this mechanism is being speculated on as a potential cause for cancer development.
\end{abstract}

\section{Contents}

1. Introduction

2. MicroRNA

3. Polyadenylation

4. Alternative polyadenylation

5. MicroRNA targets in alternative $3^{\prime}$ UTRs

6. MicroRNA targets in $C O X 2$ gene: an example

7. Switch in the use of alternative PAS, a way to cellular proliferation

8. Interplay between alternative polyadenylation and microRNA, a potential cancer link

9. Future perspectives

\section{Introduction}

As is a fact that only a small fraction $(\sim 1.5 \%)$ of the genetic material in the human genome codes for protein, most genomic DNA is non-coding but involved in the regulation

Correspondence to: Dr Xiudong Jin, Department of Pathology, Mudanjiang Medical University, Aiminqu-Tongxiangjie 3, Mudanjiang 157011, P.R. China

E-mail: xiudongjin9@126.com

Key words: microRNA, alternative polyadenylation, proliferation, cancer of gene expression $(1,2)$. Specifically, there are two levels of regulation for gene expression: transcriptional regulation controls whether a gene is transcribed or not and to what extent; post-transcriptional regulation controls the fate of the transcribed RNA molecules, including their stability, translation efficiency and subcellular localization (3). Regulation in each level requires several multi-component cellular machines, each carrying out a separate step in gene expression pathway. Instead of working as a simple linear assembly line, gene expression machines couple extensively to form a complex network to coordinate their activities, maximizing the efficiency and specificity of each step in gene expression $(4,5)$. Recent years have witnessed an increased appreciation for the importance of post-transcriptional regulation in mammalian organisms. The same primary transcript can generate a number of different isoforms by processing steps such as alternative splicing or polyadenylation (6-8). New classes of non-coding RNA genes have been described, including the abundant and conserved class of microRNAs (9-13). Alternative polyadenylation often results in mRNA 3 ' UTRs of different lengths, while microRNAs regulate gene expression by binding to specific mRNA 3' UTRs. Earlier studies have formulated the association between alternative polyadenylation and nonsense-mediated decay (14-18), A-U-rich element mediated decay (19-21) and mRNA surveillance (22), we have good reason to deduce that there should also be interplay between alternative polyadenylation and microRNA-mediated decay. In this review, we address how alternative polyadenylation and microRNAs will tether to each other for a fine tune of gene expression and its potential link to disease development, and cancer in particular.

\section{MicroRNA}

MicroRNAs are evolutionarily conserved, endogenous, singlestranded, non-coding RNA molecules of $22 \mathrm{nt}$ in length that function as post-transcriptional gene regulators (23). It has been commonly maintained that microRNAs may be able to regulate $\leq 30 \%$ of the protein-coding genes in the human genome (24), while later study indicated that $>60 \%$ of human protein-coding genes have been under selective pressure to be regulated by microRNAs (25). The mature microRNA, with the help of RNA-induced silencing complex, acts by binding to the $3^{\prime}$ UTR of target mRNAs of protein-coding genes 
and inhibiting their translation, which has several possible consequences: it can result in the cleavage of mRNA (26) or the repression of productive translation (27) or even the destabilization and reduction in the mRNA concentration by accelerating poly(A) tail removal (28).

The current consensus is that microRNA target specificity is determined by both sequence matching and target accessibility (29). The critical region for microRNA binding is nucleotides 2-8 from the 5' end of the microRNA, called the 'seed region', which binds to its target site on a given mRNA by exact Watson-Crick complementary. Asymmetry is the general rule for the matching between a microRNA and its target (30), in that the $5^{\prime}$ end of the microRNA tends to have more bases complementary to the target than the 3 ' end does. Moreover, because it is an energy consuming process to free the base pair within mRNA in order to make the target accessible for microRNA binding, secondary structures are also required for target recognition to occur. In truth, microRNA preferentially target $3^{\prime}$ UTR sites that do not have complex secondary structures and are located in accessible regions of the RNA based on favorable thermodynamics (31).

\section{Polyadenylation}

The architecture of mammalian pre-mRNA consists of coding regions and non-coding regions. The coding regions are included within a translational start codon and a stop codon, while the non-coding regions locate separately at the $5^{\prime}$ and $3^{\prime}$ end of mRNA (3). The $3^{\prime}$ end of mRNAs is generated by cleavage followed by polyadenylation (32), which is the last step of the post-transcriptional processing. Three sequence elements precisely determine the site of $3^{\prime}$ end cleavage and polyadenylation in mammalian pre-mRNAs: the poly(A) signal (highly conserved hexanucleotide AAUAAA) or its close variants, the actual polyadenylation site (PAS) 11-30 nt downstream of the signal and the G-U-rich sequence 14-70 nt downstream of the PAS (33). Mainly five protein factors are involved in the polyadenylation process: cleavage and polyadenylation specificity factor (CPSF), cleavage stimulatory factor (CstF), poly(A)polymerase (PAP), cleavage factors I and II (CFI and CFII) and poly(A)binding protein (PABP) (34). These factors bind as a complex on the precursor RNA prior to the cleavage and polyadenylation reactions. The CPSF recognizes the AAUAAA sequence and the CstF binds to the G-U-rich sequence. The CFI and CFII cleave the RNA and generate a 3 ' end for polyadenylation. The PAP performs polyadenylation through two stages with the help of PABP, adding and extending poly(A) tail to the full $\sim 200$ residue length. The 3' UTR of mature mRNA transcript runs from the stop codon to the PAS, where pre-mRNAs are polyadenylated (35).

\section{Alternative polyadenylation}

Alternative polyadenylation is defined as use of more than one polyadenylation sites, which is prevalent in at $\geq 50 \%$ of genes in mammalian genomes, producing mRNAs with different 3' UTR regions from a single transcript (36-38). At the end of the past century, Edwalds-Gilbert et al categorized alternative polyadenylation into three models, according to their distinct exon arrangement and PAS selection (35). A large number of genes have multiple PASs within the 3' UTR in the terminal exon (39), arranged one behind the other in tandem and certain regulatory elements may posit between the PASs which can influence the stability or translatability of the longer mRNA. Another set of genes have an exon which is a composite of $3^{\prime}$ and 5 ' splice sites followed closely by a PAS. Such composite exon can serve as either internal or terminal and encode different protein based on circumstances. The genes in the third model have two or more alternative 3 ' terminal exons encoding different $\mathrm{C}$-termini. They are processed into different mRNAs either by using the first 3 ' terminal exon and its PAS or by skipping over that exon entirely and using the second 3' terminal exon with its PAS. However, later researchers combined the last two models and classified alternative polyadenylation into two categories, namely splicing-independent and splicing-dependent (40). In the first case, tandem PASs are located in the same 3' exon and the UTR will consist of a constitutive part followed by an alternative part, producing mRNAs that differ solely by the length of the $3^{\prime}$ UTR. In the case of alternative terminal exons, the different $3^{\prime}$ UTR will generally not share common sequence and the choice of downstream final exon is likely coupled with suppression of the 3 ' splice site of the more upstream one, producing mRNAs that differ by their coding sequences (Fig. 1). Although both appeared as alternative $3^{\prime}$ UTRs, the functional consequences of these two types of alternative polyadenylation are obviously different. It is much easier to deduce that splicing dependent alternative polyadenylation encodes different protein products through the generation of different mRNAs $(41,42)$; while splicing independent alternative polyadenylation is more likely to generate mRNA products with differential stability, which will ultimately lead to more or less protein product, depending on enhanced or diminished RNA half-life (19,43-46).

\section{MicroRNA targets in alternative $3^{\prime}$ UTRs}

Splicing independent alternative polyadenylation produces transcript isoforms with 3' UTRs of different lengths, containing a constitutive segment and an alternative segment. If a microRNA target is located within the alternative part of the 3' UTR, the shorter transcript should be target free and should escape microRNA-mediated inhibition, while the longer transcript should be inhibited (47-49). Such variations are very common. It has been estimated that two thirds of targeted genes have alternative $3^{\prime}$ UTRs, with $40 \%$ of predicted target sites located in alternative UTR segments. Based on whether the target sites fall within constitutive and/or alternative UTR segments, Majoros and Ohler classified microRNA target genes into two broad categories (50). Constitutive targets encompass predicted target genes with constitutive UTRs, as well as genes with alternative UTRs, in which all sites are located within the constitutive UTR regions. This category accounts for $56.6 \%$ of all targets. Alternatively targeted genes have at least one target site located in an alternative 3' UTR segment, which was further, categorized into on/off targets and modulated targets. On/off targets are alternative targets in which all target sites fall exclusively into alternative UTR regions (accounting for $23.7 \%$ of all targets), whereas modulated targets contain alternative targets with sites in both constitutive and alternative UTR regions (accounting for $19.6 \%$ of all targets). This 
Splicing dependent alternative polyadenylation

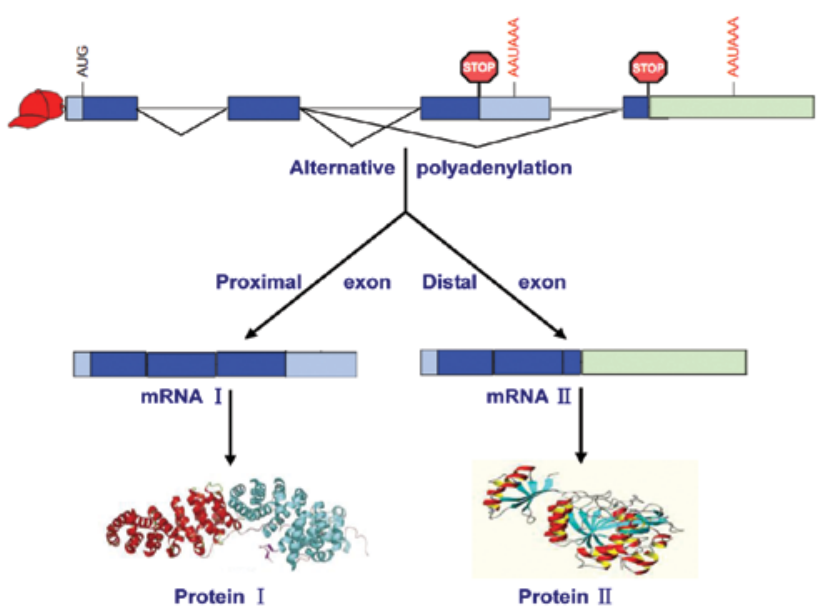

Splicing independent alternative polyadenylation

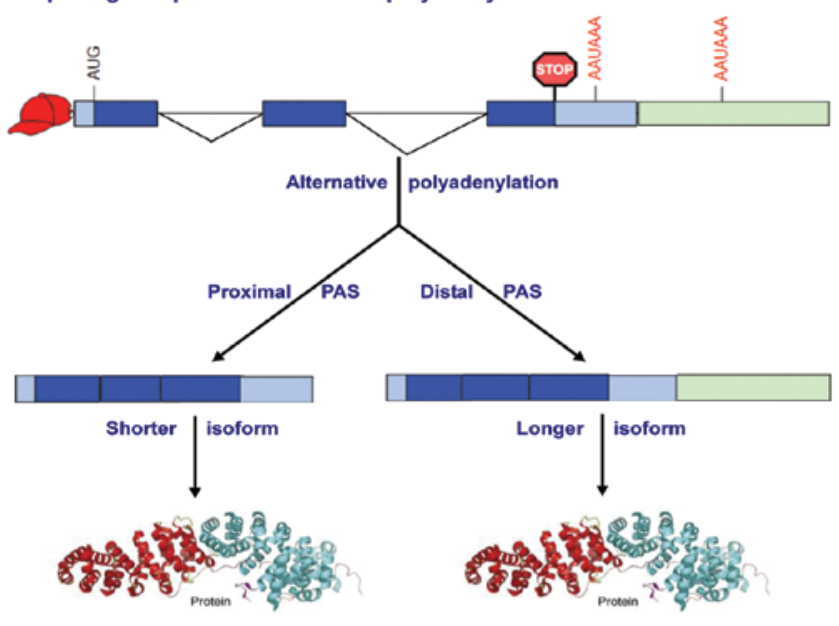

Figure 1. Splicing-dependent alternative polyadenylation and splicingindependent alternative polyadenylation.

classification of target genes perfectly illustrates the functional significance of the coupling of microRNA regulation and alternative polyadenylation. MicroRNAs regulate gene expression, while alternative polyadenylation may decide whether the expression should be regulated (on/off targets) and to what extent it should be regulated (modulated targets) (Fig. 2).

\section{MicroRNA targets in $C O X 2$ gene: an example}

Cyclooxygenases (COXs) are the key and rate-limiting enzymes in the production of prostaglandins (51). Two separate COX genes, $C O X-1$ and $C O X-2$, have been identified, divergent strikingly in their regulation of expression. COX-1 is constitutively expressed, while COX-2 is strongly induced in response to activation by hormones, pro-inflammatory cytokines, growth factors, oncogenes, carcinogens and tumor promoters (52). The $C O X-2$ gene is made up of 10 exons; the $3^{\prime} \mathrm{UTR}$ is contained within the last exon. The $C O X-23^{\prime}$ UTR is larger than average, encompassing $\sim 2.5 \mathrm{~kb}$, with several polyadenylation signals. Two of the $C O X-2$ polyadenylation signals are commonly used (53), the proximal one is AUUAAA (604-609 nt from the stop codon) and the distal one is AAUAAA (2485-2490 nt from the stop codon). The proximal signal was weaker than the distal signal and the estimated ratio of proximal/distal polyadenylation signal usage was $1: 3$. The use of the proximal polyadenylation signal results in a $\sim 2.6 \mathrm{~kb}$ mRNA, while the use of the distal polyadenylation signal results in a $\sim 4.5 \mathrm{~kb}$ mRNA. These two mRNAs were exactly the same in their coding sequences, but differ in the length of their 3' UTRs, containing a $0.6-\mathrm{kb}$ constitutive segment followed by a $1.9-\mathrm{kb}$ alternative segment (54). Within the $1.9-\mathrm{kb}$ alternative segment of $C O X-23^{\prime}$ UTR, there is a microRNA binding site (seed region 1736-1743 nt from the stop codon) for both $m i R-101$ and $m i R-199 a$. In vitro gain- and loss-of-function experiments show that $C O X-2$ expression is posttranscriptionally regulated by these two microRNAs (55). Since there is no microRNA target site confirmed in the constitutive segment of the $C O X-2$ 3' UTR, it should currently be recognized as an on/off target. The transcription of the longer isoform of $C O X-2$ mRNA will turn on the target, while the transcription of the shorter isoform will turn it off.

According to 'TargetScan' prediction, there should be two microRNA target sites within the 3' UTR of $C O X-2$. The distal target is exactly that mentioned above, within the alternative segment, while the proximal target is located in the constitutive segment (seed region 257-264 nt from the stop codon), predicted to be targeted by $m i R-26$. However, the proximal target is not experimentally verified yet. The $C O X-2$ will change from an on/off target to a modulated target depending on whether the proximal target is a true target or not. If it is a true target, then the longer isoform of $C O X-2$ mRNA will have two target sites and be regulated by two microRNAs, $m i R-26$ and $m i R-101 / m i R-199 a$, the shorter isoform will be targeted only by $m i R-26$. That is, both the long and the short isoforms will be regulated, but to different extent. In this sense, $C O X-2$ gene should modulate the extent of its regulation through switching from one isoform to the other, or by varying ratios between the two isoforms.

\section{Switch in the use of alternative PAS, a way to cellular proliferation}

The longer and the shorter isoforms of mRNA have different regulation efficiencies by microRNAs, resulting in different levels of protein expression. Will there be any preferential in the use of the isoforms under certain cellular circumstances? An interesting study (56) provides evidence that a switch in the use of alternative PASs is part of a defined program for cellular proliferation. In this study, Sandberg et al (56) developed a quantitative method to compare the expression of the constitutive segment relative to the alternative segment of the 3' UTR for genes with tandem PASs during T cell activation. The expression pattern was found to differ markedly between resting and stimulated primary T cells. Specifically, stimulation decreased the use of the alternative segment of the 3' UTR in $86 \%$ of the genes tested. This reduction was not associated with significant changes in median mRNA levels, indicating that the effect is due to a switch in PAS usage. Since activation of hematopoietic cells is often associated with a dramatic increase in proliferation, the authors hypothesized that the decrease in the use of the longer isoform, which 


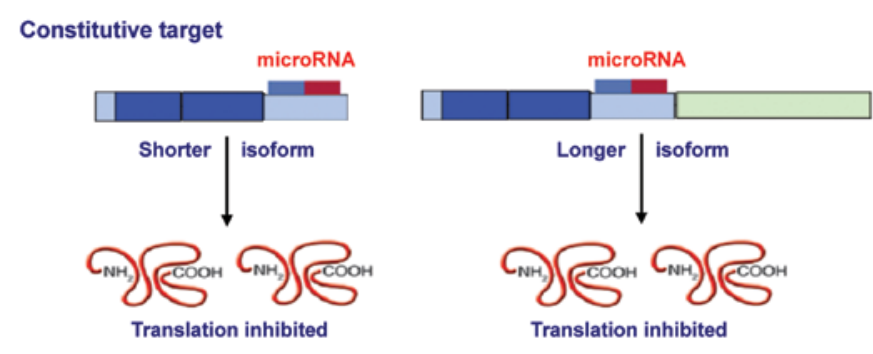

On/off target
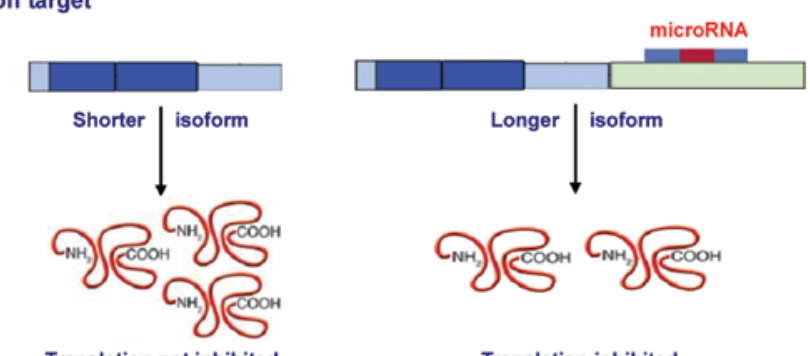

Translation not inhibited
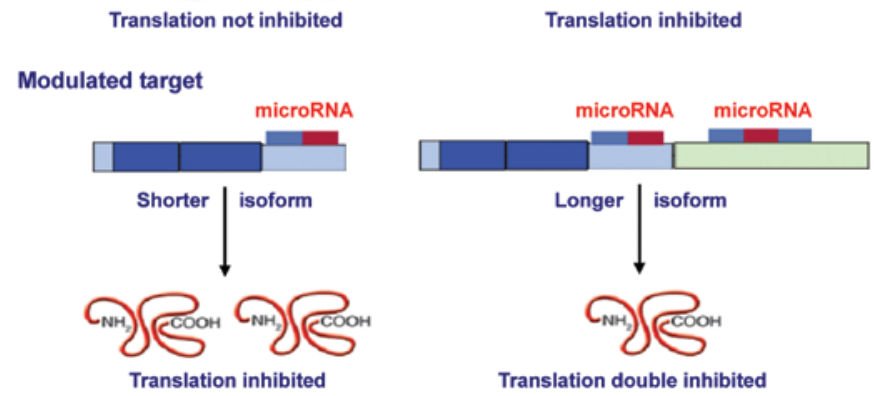

Figure 2. Three categories of microRNA targets: constitutive target, on/off target and modulated target.

leads to a shorter 3' UTR, might be associated with cellular proliferation. Interestingly, Hip2, one of the genes tested by Sandberg et al, contains in the alternative segment of its 3' UTR target sites for $m i R-21$ and $m i R-155$, both of which are expressed in activated T cells. Overall, levels of Hip2 mRNA were very similar between resting and stimulated $\mathrm{T}$ cells. However, upon $\mathrm{T}$ cell activation the relative level of the alternative segment of $3^{\prime}$ UTR decreased, while protein level increased substantially. Furthermore, elimination of the target sequences for both $m i R-21$ and $m i R-155$ from Hip 2 3' UTR increased expression level similar to that containing only the constitutive segment of 3' UTR. These results show that changes in PAS usage can control the impact of microRNA. Specifically, the use of the shorter isoform can avoid the regulation by microRNA and then enhance cellular proliferation (57).

Although it often escapes microRNA regulation, the shorter isoform of the alternatively polyadenylated mRNA is not always upregulated. In their study, Ghosh et al (58) found a microRNA mediated upregulation of the longer 3' UTR of the mouse cytoplasmic $\beta$-actin gene. This gene, also named $A c t b$, generates two alternative transcripts terminated at tandem PAS. The longer isoform was expressed at a relatively lower level, but it confered higher translational efficiency to the transcript. In fact, the longer isoform harbours a conserved binding site for $m m u-m i R-34$. Sequence specific anti-miRNA molecule, mutations of the microRNA target

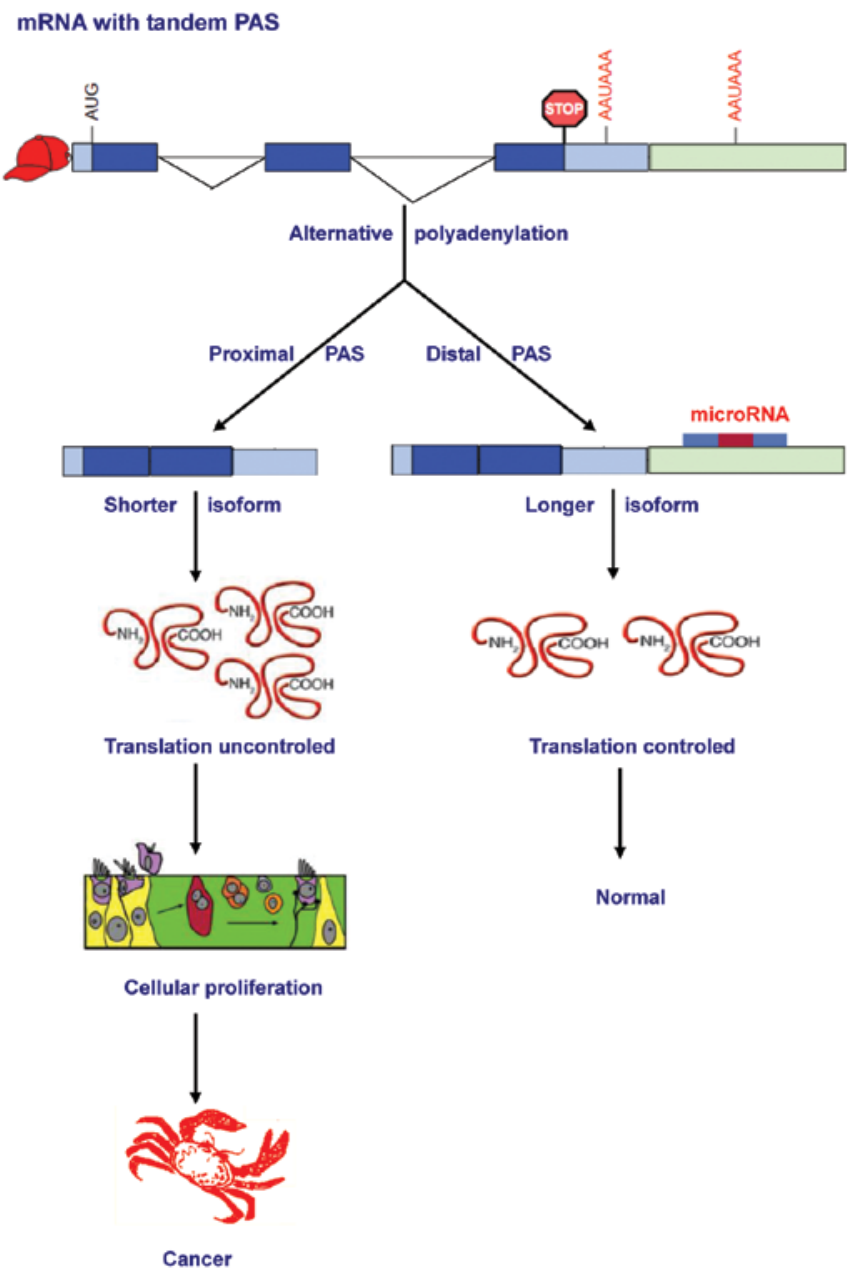

Figure 3. MicroRNAs differently regulate the longer isoform and the shorter isoform of mRNA and cause cellular proliferation and cancer.

site in the 3' UTR resulted in reduced expression and the expression could be restored by a mutant microRNA complementary to the mutated target site. These results together suggest that $m i R-34$ specifically target the longer isoform of $\beta$-actin transcript and upregulate the target gene expression, indicating that it is not always the shorter isoform that contributes to cellular proliferation.

\section{Interplay between alternative polyadenylation and microRNA, a potential cancer link}

This ability has been shown in the above-mentioned COX2 gene in colorectal cancer cells. Instead of the $4.5-\mathrm{kb}$ isoform, the 2.6-kb isoform of COX-2 mRNA lacking the miR-101/ $m i R-199 a$ target of the 3 ' UTR was selectively stabilized upon cell growth to confluence, suggesting that $C O X 2 \mathrm{mRNA}$ may escape regulation in cancer cells through alternative PAS usage (6The general association of the use of shorter 3' UTR isoforms, avoidance of regulation by microRNAs and enhanced proliferation leads to the speculation that in some cases a shift toward expression of proximal PAS isoforms may be required to evade regulation that would otherwise restrict cell cycle progression. This is in agreement with the observation that microRNAs influence many aspects 
of cellular proliferation and cell cycle progression $(59,60)$ and accordingly downregulation of microRNA expression promotes cellular transformation and tumorigenesis, which is commonly observed in cancer $(61,62)$ (Fig. 3). Many genes, under evolutionary selection, have the ability to enrich or deplete their microRNA binding sites through changes in the lengths of their 3' UTRs (30). 3). Earlier studies have shown that increased levels of COX2 protein were present in both human and animal colorectal tumors, whereas the normal intestinal mucosa displayed low to undetectable COX2 expression $(64,65)$. Similarly, elevated COX2 levels resulting from defects in its regulation was also observed in many other solid malignancies, including breast, lung, prostate, pancreas, bladder, stomach, esophagus and head and neck cancers (66-73). Therefore, it is reasonable to deduce that interplay between alternative polyadenylation of $C O X 2$ and $m i R-101 / m i R-199 a$ regulation may contribute, at least in part, to the development of cancer.

A cell cycle regulating gene (74), cyclin $D 1$ (CCND1) has two major spliced transcripts, cyclin Dla and cyclin DIb. The cyclin Dla has a wild-type isoform of $4.5 \mathrm{~kb}$ in length with a full-length $3^{\prime}$ UTR and another short isoform of 1.5 $\mathrm{kb}$ in length with a truncated $3^{\prime}$ UTR (75). Containing such a long 3' UTR, the wild-type cyclin Dla was regulated by a number of microRNAs, as indicated in certain algorisms. A regulatory feedback loop was assumed between cyclin DI and microRNA regulation, in which cyclin $D 1$ induces the expression of certain microRNAs, in turn, these microRNAs limit the proliferative function of cyclin $D 1$, thus regulating cellular tumorigenesis (76). When this feedback loop is broken, cyclin Dl will be dysregulated and overexpressed, which is commonly seen in many types of cancer, including breast, lung, colorectal cancer and lymphomas (77-80). Many strongly proliferative tumors have exceptionally high cyclin D1 mRNA levels and preferentially express short cyclin Dla mRNA isoform. This short isoform lacks many mRNA destabilization elements present in the wild-type cyclin Dla mRNA, including a target site for miR-16-1 (81). Thus, truncated cyclin Dla mRNA deletes miR-16-1 binding sites within the cyclin D1 mRNA 3' UTR and alters the microRNA regulatory feedback loop, leads to increased total cyclin D1 mRNA and increased cyclin D1 protein expression and then contributes to cancer development.

\section{Future perspectives}

By truncating their 3' UTRs, certain genes can escape the regulation of microRNAs, as well as other RNA-binding proteins and thus proliferate. Just like the gecko, breaking its tail for escape in time of danger, saving itself from being injured. This pattern of tail truncation has already been implicated in the process of disease development, including cancer. A fairly large proportion of genes have different isoforms of 3' UTR and there are full of mysteries contained in the alternative segment of the 3' UTR, besides nonsense mediated decay, A-U-rich element mediated decay and mRNA surveillance, microRNA mediated decay should be an important one. Future studies are needed to investigate the association of the interplay between alternative polyadenylation and microRNA regulation in clinical settings, which will facilitate our understanding of disease etiology and even helpful in our efforts to work out new ways of disease prevention and treatment.

\section{References}

1. Venter JC, Adams MD, Myers EW, et al: The sequence of the human genome. Science 291: 1304-1351, 2001.

2. Lander ES, Linton LM, Birren B, et al: Initial sequencing and analysis of the human genome. Nature 409: 860-921, 2001.

3. Mignone F, Gissi C, Liuni S and Pesole G: Untranslated regions of mRNAs. Genome Biol 3: Reviews 0004, 2002.

4. Maniatis T and Reed R: An extensive network of coupling among gene expression machines. Nature 416: 499-506, 2002.

5. Lackner DH, Beilharz TH, Marguerat S, et al: A network of multiple regulatory layers shapes gene expression in fission yeast. Mol Cell 26: 145-155, 2007.

6. de la Grange P, Dutertre M, Correa M and Auboeuf D: A new advance in alternative splicing databases: from catalogue to detailed analysis of regulation of expression and function of human alternative splicing variants. BMC Bioinformatics 8: 180, 2007.

7. Wang ET, Sandberg R, Luo S, et al: Alternative isoform regulation in human tissue transcriptomes. Nature 456: 470-476 2008.

8. Tian B, Pan Z and Lee JY: Widespread mRNA polyadenylation events in introns indicate dynamic interplay between polyadenylation and splicing. Genome Res 17: 156-165, 2007.

9. Hon LS and Zhang Z: The roles of binding site arrangement and combinatorial targeting in microRNA repression of gene expression. Genome Biol 8: R166, 2007.

10. Farh KK, Grimson A, Jan C, et al: The widespread impact of mammalian MicroRNAs on mRNA repression and evolution. Science 310: 1817-1821, 2005

11. Ro S, Park C, Young D, Sanders KM and Yan W: Tissuedependent paired expression of miRNAs. Nucleic Acids Res 35: 5944-5953, 2007

12. Rodriguez A, Griffiths-Jones S, Ashurst JL and Bradley A: Identification of mammalian microRNA host genes and transcription units. Genome Res 14: 1902-1910, 2004.

13. Linsley PS, Schelter J, Burchard J, et al: Transcripts targeted by the microRNA-16 family cooperatively regulate cell cycle progression. Mol Cell Biol 27: 2240-2252, 2007.

14. Kertesz S, Kerenyi Z, Merai Z, et al: Both introns and long 3'-UTRs operate as cis-acting elements to trigger nonsensemediated decay in plants. Nucleic Acids Res 34: 6147-6157, 2006.

15. Gilat R and Shweiki D: A novel function for alternative polyadenylation as a rescue pathway from NMD surveillance. Biochem Biophys Res Commun 353: 487-492, 2007.

16. Lewis BP, Green RE and Brenner SE: Evidence for the widespread coupling of alternative splicing and nonsense-mediated mRNA decay in humans. Proc Natl Acad Sci USA 100: 189-192, 2003.

17. Makeyev EV, Zhang J, Carrasco MA and Maniatis T: The MicroRNA miR-124 promotes neuronal differentiation by triggering brain-specific alternative pre-mRNA splicing. Mol Cell 27: 435-448, 2007.

18. Durand C, Roeth R, Dweep H, et al: Alternative splicing and nonsense-mediated RNA decay contribute to the regulation of SHOX expression. PloS One 6: e18115, 2011.

19. Li XL, Andersen JB, Ezelle HJ, Wilson GM and Hassel BA: Posttranscriptional regulation of RNase-L expression is mediated by the 3'-untranslated region of its mRNA. J Biol Chem 282: 7950-7960, 2007.

20. Rimokh R, Berger F, Bastard C, et al: Rearrangement of CCND1 (BCL1/PRAD1) 3' untranslated region in mantle-cell lymphomas and $\mathrm{t}(11 \mathrm{q} 13)$-associated leukemias. Blood 83: 3689-3696, 1994.

21. von Roretz $C$ and Gallouzi IE: Decoding ARE-mediated decay: is microRNA part of the equation? J Cell Biol 181: 189-194, 2008.

22. Muhlrad D and Parker R: Aberrant mRNAs with extended 3' UTRs are substrates for rapid degradation by mRNA surveillance. RNA 5: 1299-1307, 1999.

23. Bartel DP: MicroRNAs: genomics, biogenesis, mechanism, and function. Cell 116: 281-297, 2004. 
24. Filipowicz W, Bhattacharyya SN and Sonenberg N: Mechanisms of post-transcriptional regulation by microRNAs: are the answers in sight? Nat Rev Genet 9: 102-114, 2008.

25. Friedman RC, Farh KK, Burge CB and Bartel D: Most mammalian mRNAs are conserved targets of microRNAs. Genome Res 19: 92-105, 2008.

26. Yekta S, Shih IH and Bartel DP: MicroRNA-directed cleavage of HOXB8 mRNA. Science 304: 594-596, 2004.

27. Pillai RS, Bhattacharyya SN, Artus CG, et al: Inhibition of translational initiation by Let-7 MicroRNA in human cells. Science 309: 1573-1576, 2005.

28. Wu L, Fan J and Belasco JG: MicroRNAs direct rapid deadenylation of mRNA. Proc Natl Acad Sci USA 103: 4034-4039, 2006.

29. Didiano D and Hobert O: Molecular architecture of a miRNAregulated 3' UTR. RNA 14: 1297-1317, 2008.

30. Stark A, Brennecke J, Bushati N, Russell RB and Cohen SM: Animal MicroRNAs confer robustness to gene expression and have a significant impact on 3'UTR evolution. Cell 123: 1133-1146, 2005.

31. Kertesz M, Iovino N, Unnerstall U, Gaul U and Segal E: The role of site accessibility in microRNA target recognition. Nat Genet 39: 1278-1284, 2007.

32. Lopez F, Granjeaud S, Ara T, Ghattas B and Gautheret D: The disparate nature of 'intergenic' polyadenylation sites. RNA 12: 1794-1801, 2006.

33. Legendre $M$ and Gautheret D: Sequence determinants in human polyadenylation site selection. BMC Genomics 4: 7, 2003.

34. Kubo T, Wada T, Yamaguchi Y, Shimizu A and Handa H: Knockdown of $25 \mathrm{kDa}$ subunit of cleavage factor Im in Hela cells alters alternative polyadenylation within 3'-UTRs. Nucleic Acids Res 34: 6264-6271, 2006.

35. Edwalds-Gilbert G, Veraldi KL and Milcarek C: Alternative poly(A) site selection in complex transcription units: means to an end? Nucleic Acids Res 25: 2547-2561, 1997.

36. Ara T, Lopez F, Ritchie W, Benech P and Gautheret D: Conservation of alternative polyadenylation patterns in mammalian genes. BMC Genomics 7: 189, 2006.

37. Zhang H, Lee JY and Tian B: Biased alternative polyadenylation in human tissues. Genome Biol 6: R100, 2005.

38. Feng Z, Wu CF, Zhou X and Kuang J: Alternative polyadenylation produces two major transcripts of Alix. Arch Biochem Biophys 465: 328-335, 2007.

39. Moucadel V, Lopez F, Ara T, Benech P and Gautheret D: Beyond the 3' end: experimental validation of extended transcript isoforms. Nucleic Acids Res 35: 1947-1957, 2007.

40. Lutz CS: Alternative polyadenylation: a twist on mRNA 3 ' end formation. ACS Chem Biol 3: 609-617, 2008

41. Heuze-Vourc'h N, Leblond V and Courty Y: Complex alternative splicing of the hKLK3 gene coding for the tumor marker PSA (prostate-specific-antigen). Eur J Biochem 270: 706-714, 2003.

42. Liu H and Johnson EM: Distinct proteins encoded by alternative transcripts of the PURG gene, located contrapodal to WRN on chromosome 8 , determined by differential termination/polyadenylation. Nucleic Acids Res 30: 2417-2426, 2002.

43. Yu M, Sha H, Gao Y, Zeng H, Zhu M and Gao X: Alternative 3' UTR polyadenylation of Bzw1 transcripts display differential translation efficiency and tissue-specific expression. Biochem Biophys Res Commun 345: 479-485, 2006.

44. Hughes TA: Regulation of gene expression by alternative untranslated regions. Trends Genet 22: 119-122, 2006.

45. Stojic J, Stohr H and Weber BH: Three novel ABCC5 splice variants in human retina and their role as regulators of $\mathrm{ABCC} 5$ gene expression. BMC Mol Biol 8: 42, 2007.

46. Hager S, Frame FM, Collins AT, Burns JE and Maitland NJ: An internal polyadenylation signal substantially increases expression levels of lentivirus-delivered transgenes but has the potential to reduce viral titer in a promoter-dependent manner. Hum Gene Ther 19: 840-850, 2008.

47. Legendre M, Ritchie W, Lopez F and Gautheret D: Differential repression of alternative transcripts: a screen for miRNA targets. PLoS Comput Biol 2: e43, 2006.

48. Tan S, Guo J, Huang Q, et al: Retained introns increase putative microRNA targets within 3' UTRs of human mRNA. FEBS Lett 581: 1081-1086, 2007.

49. Sivakumaran TA, Resendes BL, Robertson NG, Giersch AB and Morton CC: Characterization of an abundant COL9A1 transcript in the cochlea with a novel 3' UTR: expression studies and detection of miRNA target sequence. J Assoc Res Otolaryngol 7: 160-172, 2006.
50. Majoros WH and Ohler U: Spatial preferences of microRNA targets in 3' untranslated regions. BMC Genomics 8: 152, 2007.

51. Hla T, Bishop-Bailey D, Liu CH, Schaefers HJ and Trifan OC: Cyclooxygenase-1 and -2 isoenzymes. Int $\mathrm{J}$ Biochem Cell Biol 31: 551-557, 1999.

52. Harper KA and Tyson-Capper AJ: Complexity of COX-2 gene regulation. Biochem Soc Trans 36: 543-545, 2008.

53. Hall-Pogar T, Zhang H, Tian B and Lutz CS: Alternative polyadenylation of cyclooxygenase-2. Nucleic Acids Res 33: 2565-2579, 2005.

54. Sawaoka H, Dixon DA, Oates JA and Boutaud O: Tristetraprolin binds to the 3'-untranslated region of cyclooxygenase-2 mRNA. A polyadenylation variant in a cancer cell line lacks the binding site. J Biol Chem 278: 13928-13935, 2003.

55. Chakrabarty A, Tranguch S, Daikoku T, Jensen K, Furneaux H and Dey SK: MicroRNA regulation of cyclooxygenase-2 during embryo implantation. Proc Natl Acad Sci USA 104: 15144-15149, 2007.

56. Sandberg R, Neilson JR, Sarma A, Sharp PA and Burge CB: Proliferating cells express mRNAs with shortened 3' untranslated regions and fewer microRNA target sites. Science 320: 1643-1647, 2008.

57. Zlotorynski E and Agami R: A PASport to cellular proliferation. Cell 134: 208-210, 2008.

58. Ghosh T, Soni K, Scaria V, Halimani M, Bhattacharjee C and Pillai B: MicroRNA-mediated up-regulation of an alternatively polyadenylated variant of the mouse cytoplasmic $\{$ beta\}-actin gene. Nucleic Acids Res 36: 6318-6332, 2008.

59. Gammell P: MicroRNAs: recently discovered key regulators of proliferation and apoptosis in animal cells: identification of miRNAs regulating growth and survival. Cytotechnology 53: 55-63, 2007.

60. Jovanovic $M$ and Hengartner MO: miRNAs and apoptosis: RNAs to die for. Oncogene 25: 6176-6187, 2006.

61. O'Rourke JR, Swanson MS and Harfe BD: MicroRNAs in mammalian development and tumorigenesis. Birth Defects Res C Embryo Today 78: 172-179, 2006.

62. Bandres E, Agirre X, Ramirez N, Zarate R and GarciaFoncillas J: MicroRNAs as cancer players: potential clinical and biological effects. DNA Cell Biol 26: 273-282, 2007.

63. Dixon DA: Dysregulated post-transcriptional control of COX-2 gene expression in cancer. Curr Pharm Des 10: 635-646, 2004.

64. Eberhart CE, Coffey RJ, Radhika A, Giardiello FM, Ferrenbach S and DuBois RN: Up-regulation of cyclooxygenase 2 gene expression in human colorectal adenomas and adenocarcinomas. Gastroenterology 107: 1183-1188, 1994.

65. Kutchera W, Jones DA, Matsunami N, et al: Prostaglandin H synthase 2 is expressed abnormally in human colon cancer: evidence for a transcriptional effect. Proc Natl Acad Sci USA 93: 4816-4820, 1996.

66. Ristimaki A, Sivula A, Lundin J, et al: Prognostic significance of elevated cyclooxygenase- 2 expression in breast cancer. Cancer Res 62: 632-635, 2002.

67. Wolff H, Saukkonen K, Anttila S, Karjalainen A, Vainio H and Ristimaki A: Expression of cyclooxygenase-2 in human lung carcinoma. Cancer Res 58: 4997-5001, 1998.

68. Yoshimura R, Sano H, Masuda C, et al: Expression of cyclooxygenase-2 in prostate carcinoma. Cancer 89: 589-596, 2000.

69. Tucker ON, Dannenberg AJ, Yang EK, et al: Cyclooxygenase-2 expression is up-regulated in human pancreatic cancer. Cancer Res 59: 987-990, 1999.

70. Ristimaki A, Nieminen O, Saukkonen K, Hotakainen K, Nordling S and Haglund C: Expression of cyclooxygenase-2 in human transitional cell carcinoma of the urinary bladder. Am J Pathol 158: 849-853, 2001.

71. Ristimaki A, Honkanen N, Jankala H, Sipponen $\mathrm{P}$ and Harkonen M: Expression of cyclooxygenase-2 in human gastric carcinoma. Cancer Res 57: 1276-1280, 1997.

72. Ratnasinghe D, Tangrea J, Roth MJ, et al: Expression of cyclooxygenase-2 in human squamous cell carcinoma of the esophagus; an immunohistochemical survey. Anticancer Res 19: 171-174, 1999.

73. Chan G, Boyle JO, Yang EK, et al: Cyclooxygenase-2 expression is up-regulated in squamous cell carcinoma of the head and neck. Cancer Res 59: 991-994, 1999.

74. Sherr CJ: Mammalian G1 cyclins. Cell 73: 1059-1065, 1993.

75. Wiestner A, Tehrani M, Chiorazzi M, et al: Point mutations and genomic deletions in CCND1 create stable truncated cyclin D1 mRNAs that are associated with increased proliferation rate and shorter survival. Blood 109: 4599-4606, 2007. 
76. Yu Z, Wang C, Wang M, et al: A cyclin D1/microRNA 17/20 regulatory feedback loop in control of breast cancer cell proliferation. J Cell Biol 182: 509-517, 2008.

77. Nosho K, Kawasaki T, Chan AT, et al: Cyclin D1 is frequently overexpressed in microsatellite unstable colorectal cancer, independent of $\mathrm{CpG}$ island methylator phenotype. Histopathology 53: 588-598, 2008.

78. Elsheikh S, Green AR, Aleskandarany MA, et al: CCND amplification and cyclin D1 expression in breast cancer and their relation with proteomic subgroups and patient outcome. Breast Cancer Res Treat 109: 325-335, 2008.
79. Radovic S, Babic M, Doric M, et al: Non-small cell lung carcinoma: cyclin D1, bcl-2, p53, Ki-67 and HER-2 proteins expression in resected tumors. Bosn J Basic Med Sci 7: 205-211, 2007.

80. Shakir R, Ngo N and Naresh KN: Correlation of cyclin D1 transcript levels, transcript type and protein expression with proliferation and histology among mantle cell lymphoma. J Clin Pathol 61: 920-927, 2008

81. Chen RW, Bemis LT, Amato CM, et al: Truncation in CCND1 mRNA alters miR-16-1 regulation in mantle cell lymphoma. Blood 112: 822-829, 2008. 